\title{
Towards a Mechanistic Understanding of P element Transposition Using Single- Particle Cryo-EM
}

\author{
Elizabeth H. Kellogg $2,4,5^{*}$, George Ghanim ${ }^{1,2}$, Eva Nogales ${ }^{1,2,3,4}$, and Donald C. Rio. ${ }^{1,2^{*}}$ \\ 1. Department of Molecular and Cell Biology, University of California, Berkeley, CA, USA. \\ 2. California Institute for Quantitative Biosciences, University of California, Berkeley, CA, USA. \\ 3. Howard Hughes Medical Institute. \\ 4. Lawrence Berkeley National Laboratory, Berkeley, CA, USA. \\ 5. Present address: Molecular Biology and Genetics Department, Cornell University, Ithaca, USA. \\ * Corresponding authors: don_rio@berkeley.edu and ehk68@cornell.edu
}

Transposons are mobile genetic elements that contribute to reshaping the genomes of organisms throughout the tree of life. As such, these elements are both ubiquitous and diverse. Transposons commonly encode a protein product referred to as a transposase, whose function is to mobilize the transposon utilizing either an RNA intermediate (class I) or a DNA intermediate (class II); the sequence of reactions required to complete the process is referred to as transposition. As might be expected given the diversity of transposon sequences, transposase architecture also varies widely, but some unifying structural motifs have been conserved [1]. However, these structural motifs often have low sequence similarity and are difficult to detect using sequence relationships alone, suggesting that they arise via convergent evolution. One example of such a structural motif is the RNaseH catalytic domain, with its universally conserved DDE catalytic residue triad motif (Figure 1) [2].

Single particle cryo-EM has enjoyed a resurgence of interest due to its ability to obtain high-resolution structural information on challenging samples. Examples of intrinsically disordered proteins [3], low abundance macromolecular complexes [4-5], and aggregation-prone samples [6] such as HIV integrase [7] have recently been solved to a resolution of $4 \AA$ or better. This resolution is sufficient to infer the atomic structure from the cryo-EM density map, specially when aided by molecular modelling [8-9]. Rapid advances in cryo-EM data acquisition and image processing make it now possible to overcome historically crippling and common problems, such as the preferential orientation problem, by modifying data acquisition (i.e. collecting data from tilted specimens) [10].

One particular class of transposable element, called the $\mathrm{P}$ element, encodes a transposase with unique mechanistic features, distinct from other members of the DDE superfamily. Among these features of the $\mathrm{P}$ element transposase (TNP) are the generation of unusually long (17nt) staggered cuts upon excision from its host genome, and the unique requirement for the nucleotide cofactor guanosine triphosphate (GTP) to carry out transposition [11-12]. Due to its sequence divergence, the P element transposase RNAseH domain and catalytic residues remains hypothetical $[2,13]$, and most of the structure remains unresolved. We are using high-resolution cryo-EM (Figure 2) to obtain a structure of the P element transposase [14].

References:

[1] SP Montaño, YZ Pigli and PA Rice, Nature 491 (2012), p. 413.

[2] YW Yuan and SR Wessler, PNAS 108 (2011), p. 7884.

[3] EH Kellogg et al., Science 360 (2018), p. 1242. 
[4] AB Patel et al., Science 362 (2018).

[5] BJ Greber et al., Nature 549 (2017), p. 414.

[6] A Ballandras-Colas et al., Science 355 (2017), p. 93.

[7] DO Passos et al., Science 355 (2017), p. 89.

[8] Frenz, Nature Methods 14 (2017), p. 797.

[9] PV Afonine et al., PD Acta Cryst D. Struct. Biol 74 (2018), p. 531.

[10] YZ Tan et al., Nature Methods 14 (2017), p. 793.

[11] PD Kaufman and DC Rio, Cell 69 (1992), p. 27.

[12] M Tang et al., Genes Dev 19 (2005), p. 1422.

[13] Majumdar and DC Rio, Microbiol. Spectr 2 (2015), MDNA3-0004-2014.

[14] Authors EHK and GG contributed equally.

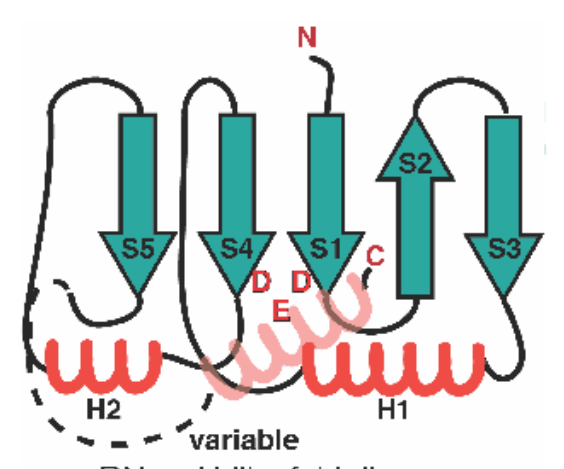

RNaseH-like fold diagram

Figure 1. Diverse transposase architectures contain common structural motifs, such as the RNAseH catalytic domain

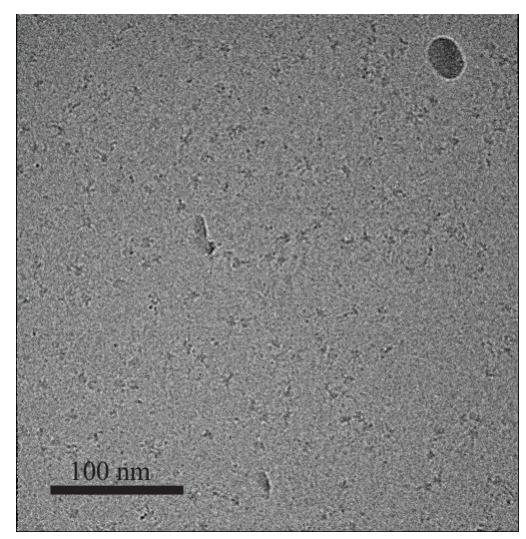

Figure 2. A representative cryo-EM micrograph of $\mathrm{P}$ element transposase 